\title{
A Young Male with Recurrent Chest Infections and Clubbing
}

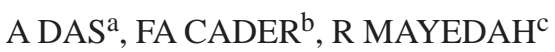

A 25 year old Bangladeshi gentleman presented with fever, cough with productive sputum and breathlessness for seven days. Fever was intermittent, high grade associated with chills and rigors, highest recorded temperature as 101 degrees Fahrenheit. Sputum was yellowish, copious in volume, foul-smelling but not associated with haemoptysis. He also complained of rhinitis and intermittent headaches. On query, he admitted to recurrent episodes of common cold, sneezing and nasal blockade, and productive cough with exertional breathlessness for the past 8 years. He was a non-smoker, and born to non-consanguineous parents. He had been treated repeatedly with various antibiotics and expectorant medication for his condition with no success.

On examination, he had stable haemodynamic parameters and digital clubbing. Lung auscultation revealed vesicular breath sounds with prolonged expiration, and bi-phasic coarse crackles and wheeze over both lung fields. Precordial examination revealed dextrocardia, with apex beat in the left fifth intercostal space; heart sounds were both audible in the right side of the chest with no murmurs. As he was unmarried and denied sexual activity it was difficult to elicit a history of infertility.

Chest X-ray PA view revealed cardiac apex and aortic arch on the right side, suggesting dextrocardia; fundic gas shadow was on the right side, indicating situs

a. Dr. Aparna Das, Associate Professor, Department of Medicine, Dhaka Medical College Hospital, Dhaka

b. Dr. F. Aaysha Cader, Honorary Medical Officer, Department of Medicine, Dhaka Medical College Hospital, Dhaka.

c. Dr. Rita Mayedah, Honorary Medical Officer, Department of Medicine, Dhaka Medical College Hospital, Dhaka.

Address of Correspondence: Dr. Aparna Das, Associate Professor, Department of Medicine, Dhaka Medical College Hospital, Dhaka. Email: aparna_0191@yahoo.com inversus; there was also evidence of bilateral with ring shadows suggestive of bronchiectasis (Fig-1) .

Electrocardiogram showed evidence of dextrocardia with right axis deviation, positive QRS complexes with upright $\mathrm{P}$ and $\mathrm{T}$ waves in lead aVR, inverted $\mathrm{P}$ and $\mathrm{T}$ wave inversion in lead $\mathrm{I}$, and absent $\mathrm{R}$ wave progression of the chest leads (Fig - 2). HRCT of chest showed ring shadows of thickened walls of dilated bronchi more predominant over the basal lung fields indicate bilateral bronchiectasis (Fig -3) .Xray of the paranasal sinuses occipito-mental view revealed bilateral maxillary sinusitis (Fig - 4). Blood counts revealed mild leucocytosis. Sputum culture revealed no growth. Sputum for AFB not found. MT negative. Our patient was unmarried and denied any sexual activity so it was difficult to come to a conclusion regarding his fertility,

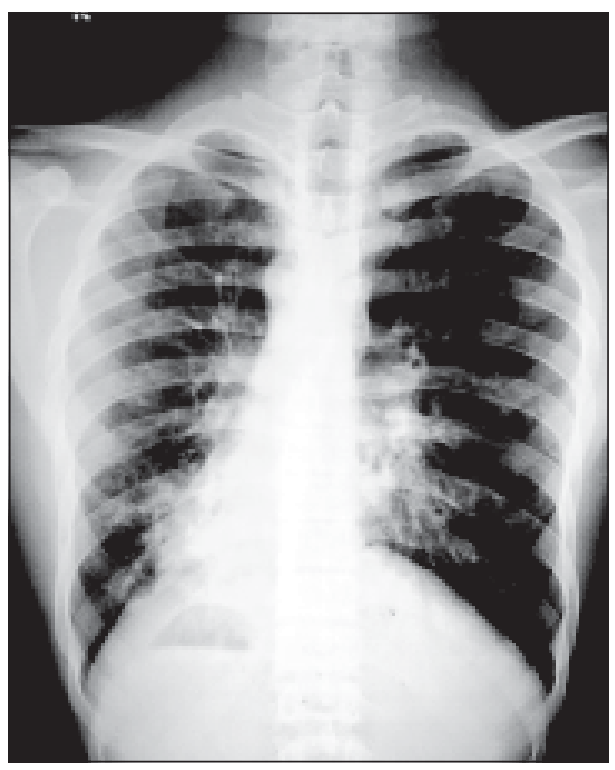

Fig.-1 : Chest Xray PA view showing dextrocardia, fundal gas shadow on the right side, and evidence of bilateral bronchiectasis. 


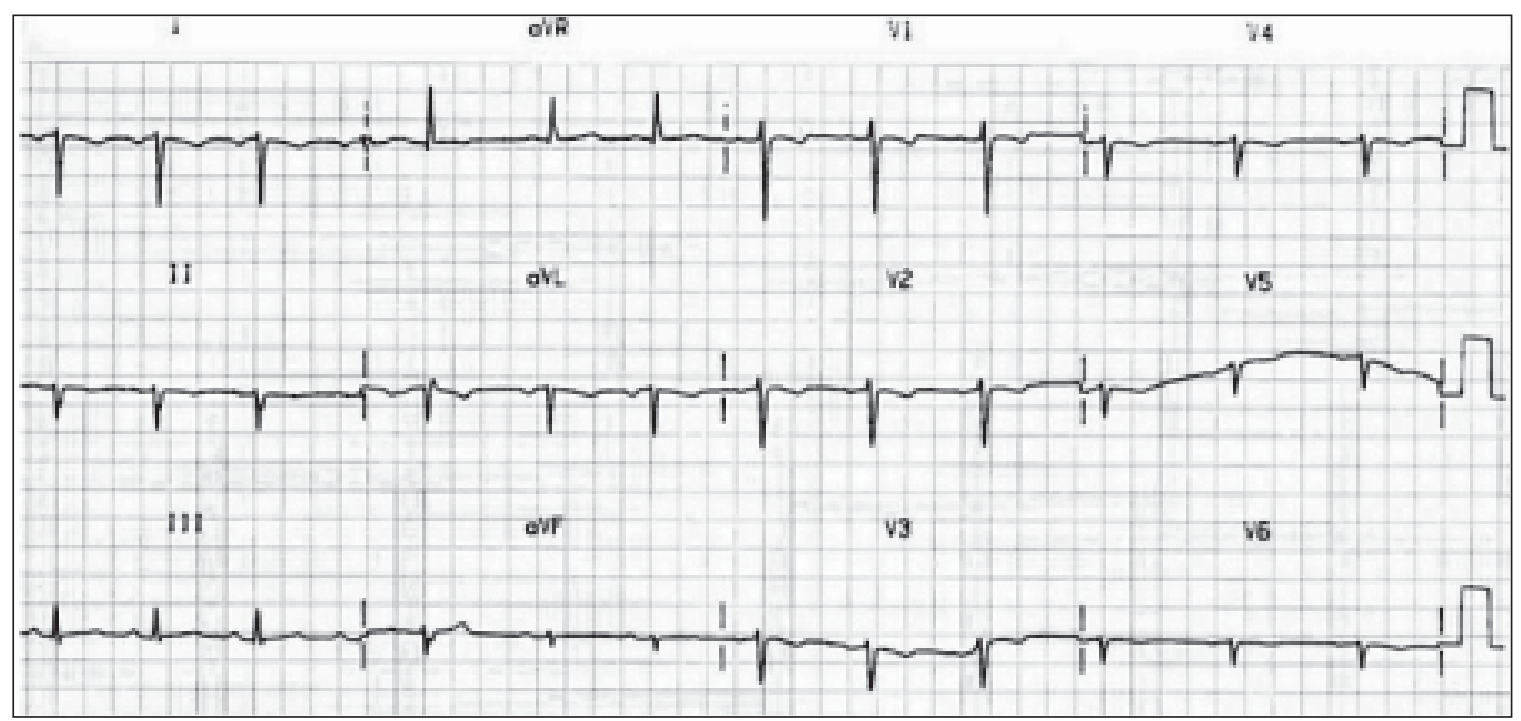

Fig.-2 ECG tracing showing dextrocardia

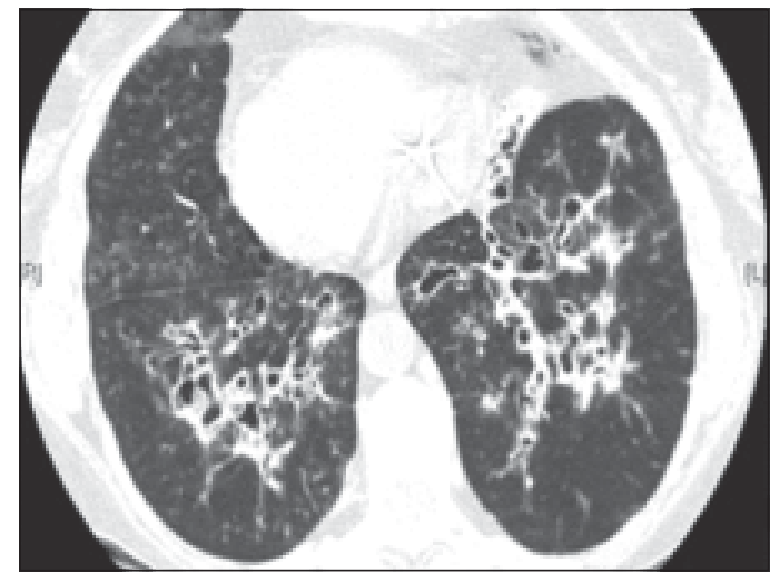

Fig.-3 : HRCT of chest showing dextrocardia and ring shadows of thickened walls of dilated bronchi more predominant over the basal lung fields indicate bilateral bronchiectasis

but he counselled regarding the possibility of infertility and the need for semen analysis. The patient was given chest physiotherapy, bronchodilators and intravenous antibiotics for 7 days. He was afebrile and showed clinical improvement. The patient was discharged on immunomodulatory therapy comprising of Azithromycin thrice weekly.

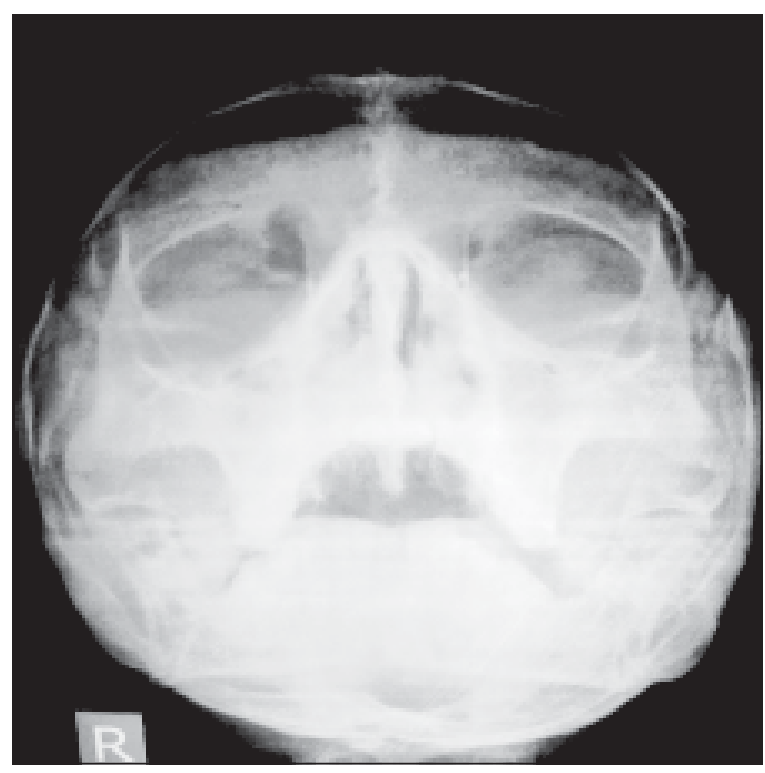

Fig- 4 : Xray of the paranasal sinuses occipito-mental view showing bilateral maxillary sinusitis

\section{Discussion:}

Kartagener's syndrome (KS), first described in 1933 by Kartagener, ${ }^{1}$ is a rare, autosomal recessive genetic disorder comprising a triad of situs inversus, bronchiectasis and sinusitis. ${ }^{1-3}$ It is a subset and the most severe form of a larger group of ciliary motility disorders called primary ciliary dyskinesias (PCDs), previously 
known as Immotile Cilia Syndrome. ${ }^{4}$ PCD, with an estimated prevalence of 1 in 20,000 to 30,000, manifests early in life and that distinguishes it from the acquired mucociliary disorders. ${ }^{5}$ The gene defect results in impaired ciliary motility of the cilia lining the mucosa of the respiratory tract and sinus, leading to recurrent chest infections, bronchiectasis, rhino- sinusitis and otitis media early on life; ${ }^{2-3}$ in addition, infertility has been reported later in life resulting from immotile sperms. ${ }^{5}$ In females, subfertility is due to defective ovum transport due to dyskinetic motion of oviductal cilia. ${ }^{2}$ Situs inversus can be seen in about $50 \%$ of cases. ${ }^{3,5}$

Diagnostic criteria for KS include ${ }^{6}$ clinical picture suggestive of recurrent chest infections, bronchitis, and rhinitis since childhood, along with one or more of the following: (1) situs inversus in the patient/sibling; (2) alive but immotile spermatozoa; (3) reduced or absent trans bronchial mucociliary clearance; and (4) cilia showing characteristic ultrastructural defect on electron microscopy. ${ }^{2,6}$ Our patient fulfil the criteria of KS.

Apart from fulfilling the criteria, diagnosis can be made by tests to prove impaired cilia function, biopsy, and genetic studies. ${ }^{3,7}$ Measuring exhaled nasal nitric oxide which is mostly reduced or absent is a good screening test for PCD with a good negative predictive value. ${ }^{3}$ Saccharin test to assess mucociliary function of nasal epithelium can also be done. ${ }^{2}$ Electron microscopy of a nasal or bronchial biopsy may reveal defected cilia structure. ${ }^{3}$ Evaluation of sperm motility can help make the diagnosis in cases where other test results are equivocal. Owing to lack of facilities, we could not perform these tests in our case, and the diagnosis was essentially clinico-radiological. In fact, the diagnosis can be easily made clinically and supported by basic imaging.

The most common respiratory pathogens are Haemophilusinfluenzae and Streptococcus pneumoniae. ${ }^{5}$ Pseudomonas has also been reported, in fatal cases. ${ }^{3}$ Hence antibiotic therapy should be tailored according to sputum culture and sensitivity patterns. Clinical progression of the disease is variable with lung transplantation required in severe cases. ${ }^{2}$
Prognosis can be fatal in severe cases if bilateral lung transplantation is delayed. ${ }^{3}$

Treatment of KS includes daily chest physiotherapy, antibiotics and supportive pulmonary care. DNase and other mucolytic agents such as hypertonic saline and acetylcysteine may be tried. Surgical intervention for bronchiectasis is rarely recommended, but can be beneficial when the disease is localized. ${ }^{3}$ Timely treatment options such as subzonal insemination (SUZI) and intracytoplasmic sperm injection (ICSI) also need to be offered for infertility. ${ }^{2}$

\section{Conclusion:}

As KS tends to present with fairly common symptoms such as copious productive cough and breathlessness, it could very often be misdiagnosed as tuberculosis especially in the Indian subcontinent; in fact there have been some case reports where patients have received long courses of anti_TB treatement with no relief, also resulting in a delayed diagnosis of KS. ${ }^{2}$

Ahigh index of suspicion is required for the diagnosis of this condition, and to look for other components of the triad. Early diagnosis is particularly important for prompt commencement of treatment in order to preserve pulmonary function and life expectancy.

\section{References:}

1. Kartagener M, Horlacher A. Situsvisceruminversus and polyposisnasi in eineumFalle familiaererBrouchiectasien. BeitrKlin Tub 1936; 87: 331-33

2. Mishra M, Kumar N, Jaiswal A, Verma AK, Kant S. Kartagener's syndrome: A case series. Lung India/ : Official Organ of Indian Chest Society2012;29(4):366-369.

3. Skeik N, Jabr FI. Kartagener syndrome.Int J Gen Med. 2011; 4: 41-43.

4. Bhatia MS,Bhasin C, Bansa S. An atypical case of Primary Ciliary Dyskinesia (Kartagener Syndrome) Delhi Psychiatry Journal 2008; 11:(1)

5. Bavaliaya M, Sadatia V, Vekariya R, Jani D. Kartagener'sSyndrome : A Rare Case. NJIRM 2012; 3(4) : 139-141

6. Afzelius BA, Mossberg B. Immotile cilia. Thorax. 1980; 35: 401-4.

7. Stannard WA, Chilvers MA, Rutman AR, Williams CD, O’Callaghan C. Diagnostic testing of patients suspected of primary ciliary dyskinesia. Am J Respir Crit Care Med. 2010;181(4):307-314. 